\title{
Thermal Environment inside a Tunnel after Thermobaric Explosion
}

\author{
Fei Chen, Jinfeng Mao, Jin Zhou, Pumin Hou, Liyao Liu, and Shangyuan Chen
}

College of Defense Engineering, PLA University of Science \& Technology, Nanjing 210007, China

Correspondence should be addressed to Jinfeng Mao; maojinfeng628@163.com

Received 3 February 2017; Revised 8 May 2017; Accepted 11 June 2017; Published 19 July 2017

Academic Editor: Isabelle Sochet

Copyright (c) 2017 Fei Chen et al. This is an open access article distributed under the Creative Commons Attribution License, which permits unrestricted use, distribution, and reproduction in any medium, provided the original work is properly cited.

\begin{abstract}
The outstanding thermal damage effect of thermobaric explosive (TBX) is enhanced in closed or semiclosed spaces, which may pose a serious threat to the security of people sheltered in tunnels or other protective engineering. In order to investigate the thermal environment inside a tunnel after thermobaric explosion, we developed a damage evaluation method for the thermal radiation of explosion fireballs in tunnels; secondly, the air temperature distribution inside a tunnel shortly after explosion was theoretically analyzed; finally, the dynamic thermal environment after the explosion and the influences of TBXs mass and initial ground temperature on it in cases of open and blocked tunnels were numerically simulated with the FLUENT software. The results show that the fireball thermal radiation damage occurs mainly in the vicinity of the explosion source. The air temperature inside a tunnel shortly after the explosion decreases continuously with increasing distance from the explosion source and finally reaches the initial air temperature. The decay rate of air temperature inside a tunnel is slower in the blocked case, which increases the probability of causing a secondary fire disaster. The increase of explosive mass and the initial ground temperature favor the high-temperature performance of TBX, especially for the blocked tunnel.
\end{abstract}

\section{Introduction}

Thermobaric explosive (TBX), a new subcomponent of volumetric explosives presents a number of advantages with respect to traditional high explosives, including longer duration, higher impulse shock wave, and diversified damage effects $[1,2]$. The weapons filled with TBX depend mainly on the pressure and thermal effects instead of armour-penetrating or fragmentation damage effects to achieve destruction, which overcomes the shortcomings of typical blast/fragmentation munitions for specific targets, such as buildings, field fortifications, and tunnels [3-5]. Therefore, TBX is a serious threat to the survival of existing protective engineering in wartime.

Due to its outstanding features, the damage effects of TBX have been the focus of much research in recent years. Many studies have been conducted on the overpressure field and pressure damage effects [6-10], while research on thermal effects is less because of the inherent view that the damage severity of shock wave is far greater than that of thermal radiation. This view is always correct for traditional high explosives, but not for TBX, since the addition of high energy metal powder makes the reaction time and space scale of TBX higher than those of high explosives. In some closed, semiclosed, and other specific circumstances, the explosion thermal damage severity even exceeds the shock wave [11]. Therefore, it is of great significance to study the thermal effects of TBX, including explosion fireball radiation and dynamic thermal environment after explosion in adjacent areas of explosion source.

Mohamed et al. [12] recorded the explosion events in an open field for a novel thermobaric formulation with $2 \mathrm{~kg}$ using the high speed camera recorder; the results showed that the mushroom shape and extended action of fireball are two main TBX characteristics, and the effective lethal fireball duration can be up to $50 \mathrm{~ms}$. The work of Yan et al. [13] has come to similar conclusions. Li and Hui [14] investigated the detonation temperature of TBX using infrared thermal image instrument and found that the duration of high temperature and the volume of the high-temperature cloud were $2 \sim 5$ and 2 10 times as much as those of TNT, respectively, which implies that TBX is superior to the traditional high explosive on the temperature field. 
Combined with certain fireball models and thermal damage criterion, the damage range of thermal radiation can be evaluated. Guo et al. [15] measured the blasting fireball's temperature of TNT and TBX with different mass and then calculated the thermal damage radius of them combined with Baker's fireball model; the results showed that TBX possesses much better thermal damage effects than TNT. On the basis of their work, Li et al. [16] studied the thermal sustaining damage ability of TBX by using the thermal radiation damage equation of Pietersen, which takes into account the influence of explosion duration, as the criterion of heat damage effect and the results showed that TBX has a stronger hightemperature sustaining damage capacity. Zhong et al. [17] analyzed the thermal damage of TBX and TNT fireballs based on the Martinsen dynamic model, which can describe the dynamic changes of fireball; the results showed that the heat dose of TBX was 3.6 4.8 times as much as that of TNT.

The above studies were conducted in the open field and cannot well reflect the thermal damage effects of TBX because of the shorter duration of fireballs. In terms of closed or semiclosed environment explosion, there are several studies. Using an enclosed explosion container to simulate the limited space, Yan et al. [18] measured the explosion field temperature of TBX to investigate the influence of postburn effect and the results showed that the effect could obviously increase the temperature of explosive products and make it keep at high level for a long time. Yan et al. [19] studied the thermal effect of TBX under semiclosed condition by testing the thermal response in tunnel after explosion using thermocouples and the results showed that the temperature field of TBX has wider range, higher temperature, and longer duration in tunnel condition, compared with that of TNT with the same mass.

However, few studies have been done on the thermal radiation evaluation method of thermobaric explosion in closed or semiclosed spaces, and even fewer works are available on the dynamic thermal environment after the explosion in such spaces, due to the difficulty in conducting effective measurements in such poor test conditions and with limited testing methods. In this paper, we first establish a damage evaluation method for the thermal radiation of thermobaric explosion fireball in a tunnel and then theoretically deduce the air temperature distribution inside a tunnel shortly after the explosion. Taking this distribution as an initial condition, we analyze the dynamic thermal environment after the explosion and the influences of TBXs mass and initial ground temperature on it by simulating the temporal and spatial variation of air temperature in a tunnel with the FLUENT software. The purpose of this paper is to investigate the thermal environment inside a tunnel after thermobaric explosion and provide prerequisites for thermoprotection design of protective engineering.

\section{Thermal Radiation Damage Evaluation}

In order to evaluate the thermal radiation damage effects of a thermobaric explosion fireball, it is first necessary to determine the heat flux or heat dose received by the targets surface. However, it is often difficult to achieve an accurate experimental measurement of these two parameters. To solve this problem, various types of thermal radiation models for fireballs, suitable for different fuels or explosives, have been proposed, including the Dorofeev model [20], the Baker model [21], and the Martinsen model [22]. The Baker model is a kind of semiempirical, universal, and static model, whose applicability to TBX has been demonstrated [15]. Therefore, this model is chosen for the present study.

In the Baker model, the heat flux and heat dose received by the targets surface can be, respectively, expressed according to the following two equations [21]:

$$
\begin{aligned}
\frac{q}{T_{F}^{4}} & =\frac{G\left(D_{F}^{2} / L^{2}\right)}{F+D_{F}^{2} / L^{2}}, \\
\frac{Q}{(b G) m^{1 / 3} T_{F}^{2 / 3}} & =\frac{D_{F}^{2} / L^{2}}{F+D_{F}^{2} / L^{2}},
\end{aligned}
$$

where $q$ is the heat flux; $Q$ is the heat dose; $T_{F}$ is the temperature of the fireball; $D_{F}$ is its diameter; $L$ is the distance from the center of the fireball; $m$ is the mass of consumed fuel, which in this case can be taken as the mass of thermobaric charge here; $F$ is a constant whose value is $161.7 ; b G$ is another constant whose value is $2.04 \times 10^{4}$.

The heat dose damage criterion is chosen in this paper to evaluate the thermal radiation damage effects of a thermobaric explosion fireball due to its applicability to a variety of transient combustion or explosion processes [15]. The corresponding damage threshold values are shown in Table 1.

For the thermobaric explosion in an open space, the thermal radiation damage range can be calculated by

$$
L=D_{F} \sqrt{\frac{(b G) m^{1 / 3} T_{F}^{2 / 3}-Q}{Q F}} .
$$

However, when the TBX explodes inside a tunnel, the flame will be constrained by the surrounding rocks, which is obviously a different process from the fireball generated by an open space explosion. In order to apply Baker model, which is suitable for open space explosions only, to the confined space explosion, the concept of the tunnel equivalent heat dose $Q_{T}$ is proposed based on the following assumptions:

(1) The explosion process is instantaneous, and the heat damage is caused by the fireball radiation.

(2) The effects of heat conduction, heat convection, and the heat radiation absorbed by air and by the surrounding rocks are ignored.

(3) The tunnel is straight with a uniform section, surrounded by smooth and adiabatic rocks.

The relationship between the tunnel equivalent heat dose and the open space heat dose can be expressed as

$$
4 \pi\left(\frac{D_{F}}{2}\right)^{2} \cdot Q=2 S \cdot Q_{T},
$$

namely,

$$
Q=\frac{2 S \cdot Q_{T}}{\pi D_{F}^{2}},
$$


TABLE 1: Thermal radiation damage range for the thermobaric explosion in a tunnel.

\begin{tabular}{lcc}
\hline Damage effects & $\begin{array}{c}\text { Damage threshold } \\
\text { values }\left(\mathrm{kJ} / \mathrm{m}^{2}\right)[23]\end{array}$ & Damage range (m) \\
\hline Igniting wood & 1030 & 30.14 \\
Death & 592 & 39.77 \\
Serious injury & 392 & 48.88 \\
Third-degree burn & 375 & 49.97 \\
Second-degree burn & 250 & 61.21 \\
Slight injury & 172 & 73.80 \\
First-degree burn & 125 & 86.56 \\
Cutaneous pain & 65 & 120.05 \\
\hline
\end{tabular}

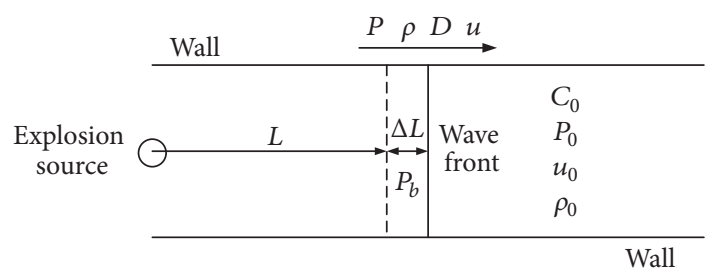

FIGURE 1: Schematic illustration of the plane shock wave propagation.

where $S$ is the cross-sectional area of the tunnel; $Q_{T}$ is the tunnel equivalent heat dose, which can be set to different damage threshold values in Table 1.

Substituting (4) into (2), the thermal radiation damage range for the thermobaric explosion in a tunnel can be calculated by

$$
L_{T}=D_{F} \sqrt{\frac{0.5 \pi(b G) m^{1 / 3} T_{F}^{2 / 3} D_{F}^{2}-S Q_{T}}{S Q_{T} F}} .
$$

Letting $m=100 \mathrm{~kg}, S=4.32 \mathrm{~m}^{2}, T_{F}=3153.31 \mathrm{~K}$, and $D_{F}=11.96 \mathrm{~m}$, the calculated thermal radiation damage range is listed in Table 1.

\section{Temperature Distribution}

3.1. Modeling. When the explosive shock wave propagates in a straight tunnel, it will gradually form a stable plane shock wave after undergoing several reflections [24]. In order to study the temperature distribution shortly after the thermobaric explosion, a plane shock wave propagation model is established as shown in Figure 1.

The following assumptions have been made in order to simplify the model:

(1) Heat transfer and friction loss during the shock wave propagation are ignored, and the process is considered adiabatic.

(2) The gas state in the whole process satisfies the ideal gas equation.

(3) All the explosion energy of TBX has been released before the formation of the plane shock wave, ignoring the chemical reaction process. Moreover, the explosion energy is constant for a certain amount and type of thermobaric charge.

(4) The amount of the gas compressed by the shock wave is concentrated in a very thin layer of thickness $\Delta L$, immediately adjacent to the shockwave front. The gas density distribution in the thin layer is considered as uniform and equal to the wave front gas density $\rho$. The gas mass in the thin layer equals to the total mass of gas existing in the tunnel of length $L$ and cross-sectional area in $S$ before the perturbation of the shock wave according to the following equation:

$$
m_{b}=\Delta L S \rho=L S \rho_{0} .
$$

(5) The flow velocity distribution in the thin layer is uniform and equal to the wave front velocity $u$.

(6) The pressure distribution in the thin layer is uniform and equal to the wave front pressure $P$ multiplied by an undetermined parameter $\alpha$.

According to the momentum theorem, the gas in the thin layer must satisfy the following relation:

$$
S\left(P_{b}-P_{0}\right) d t=S\left(\alpha P-P_{0}\right) d t=d\left(m_{b} u\right),
$$

where $P=(2 /(\gamma+1)) \rho_{0} D^{2}\left(1-C_{0}^{2} / D^{2}\right)+P_{0} ; u=(2 /(\gamma+$ 1)) $D\left(1-C_{0}^{2} / D^{2}\right)$.

Using the relations $P_{0}=C_{0}^{2} \rho_{0} / \gamma$ and $d / d t=(d / d L)(d L /$ $d t)=D(d / d L)$, (8) can be obtained by integrating (7).

$$
D \sqrt{1+\frac{1-\gamma}{2 \gamma} \frac{C_{0}^{2}}{D^{2}}}\left(1+\frac{1-\gamma}{2 \gamma} \frac{C_{0}^{2}}{D^{2}}\right)^{\gamma /(\gamma-1)}=A L^{\alpha-1} .
$$

For a very strong shock wave, $C_{0}^{2} / D^{2} \rightarrow 0$. Therefore, the above equation can be simplified as

$$
D=A L^{\alpha-1},
$$

where $A$ is an undetermined parameter.

According to assumption (1), the energy released by the TBX explosion is fully converted into work, increasing the internal energy and kinetic energy of the thin layer gas. The internal energy can be expressed as

$$
E_{b}=\frac{S L P_{b}}{\gamma-1} .
$$

The kinetic energy can be expressed as

$$
E_{b k}=\frac{1}{2} m_{b} u^{2} .
$$

The explosion of thermobaric charges is a process which can be divided into an anaerobic stage and aerobic stage (or postcombustion stage). The energy released in the anaerobic stage is known as explosion heat $Q_{V}$, and the energy released in the aerobic stage, when the reaction products are stable, is referred to as combustion heat $Q_{L}$. Due to the limited support of the energy released in the postcombustion stage to the 
detonation [25], the explosion heat is used as the explosion released energy. Taking into account the actual situation, a safety factor of 1.2 is employed to reduce the error caused by ignoring the energy loss and the reaction of the aluminum powder. According to the law of conservation of energy, we have the following balance:

$$
1.2 m Q_{V}=E_{b}+E_{b k}=\frac{S L P_{b}}{\gamma-1}+\frac{1}{2} m_{b} u^{2}
$$

where $Q_{V}$ is the explosion heat of TBX; $m$ is the mass of thermobaric charge. Equation (12) can be further derived as

$$
1.2 m Q_{V}=2 A^{2} S \rho_{0}\left(\frac{\alpha}{\gamma^{2}-1}+\frac{1}{(\gamma+1)^{2}}\right) L^{2 \alpha-1} .
$$

According to the assumption (3), $Q_{V}$ and $L$ are irrelevant for a certain amount and type of thermobaric charge. Therefore, $\alpha=0.5$ can be obtained by solving from the above equation. By substituting this value in (13) and solving for $A$, we obtain

$$
A=\sqrt{\frac{(\gamma-1)(\gamma+1)^{2} 1.2 m Q_{V}}{(3 \gamma-1) S \rho_{0}}} .
$$

Therefore, (9) can be expressed as

$$
D=\sqrt{\frac{(\gamma-1)(\gamma+1)^{2} 1.2 m Q_{V}}{(3 \gamma-1) S \rho_{0}}} L^{-0.5} .
$$

Combined with the basic relation of a strong shock wave, which is $P=(2 /(\gamma+1)) \rho_{0} D^{2}$, the peak overpressure distribution can be expressed by the following equation:

$$
\Delta P=\frac{2.4\left(\gamma^{2}-1\right) m Q_{V}}{(3 \gamma-1) S} \frac{1}{L}=\frac{2.4\left(\gamma^{2}-1\right) Q_{V}}{3 \gamma-1}\left(\frac{m}{S L}\right) \text {. }
$$

According to assumption (2), we have $P=\rho R T$. Therefore, the relation between the air temperatures at the front and back of the shock wave can be expressed as

$$
\begin{aligned}
T & =T_{0} \frac{\rho_{0}}{\rho} \frac{P}{P_{0}}=T_{0} \frac{\rho_{0}}{\rho} \frac{\Delta P+P_{0}}{P_{0}}=T_{0} \frac{\rho_{0}}{\rho}\left(1+\frac{\Delta P}{P_{0}}\right) \\
& =T_{0}\left(1+\frac{\Delta P}{P_{0}}\right) \frac{1+((\gamma-1) / 2 \gamma)\left(\Delta P / P_{0}\right)}{1+((\gamma+1) / 2 \gamma)\left(\Delta P / P_{0}\right)},
\end{aligned}
$$

where $T, \rho$, and $P$ are the temperature, density, and pressure of the air behind the shock wave, respectively; $T_{0}, \rho_{0}$, and $P_{0}$ are the temperature, density, and pressure of the air at the front of the shock wave, respectively; $\Delta P$ is the peak overpressure; $\gamma$ is the specific heat ratio, which is equal to 1.4 for air.

Substituting (16) into (17), we obtain the theoretical expression of the air temperature distribution in the tunnel shortly after the thermobaric explosion:

$$
\begin{aligned}
T= & T_{0}\left[1+\frac{2.4\left(\gamma^{2}-1\right) Q_{V}}{P_{0}(3 \gamma-1)}\left(\frac{m}{S L}\right)\right] \\
& \cdot \frac{2 \gamma(3 \gamma-1) P_{0}+2.4(\gamma+1)(\gamma-1)^{2} Q_{V}(m / S L)}{2 \gamma(3 \gamma-1) P_{0}+2.4(\gamma-1)(\gamma+1)^{2} Q_{V}(m / S L)}
\end{aligned}
$$

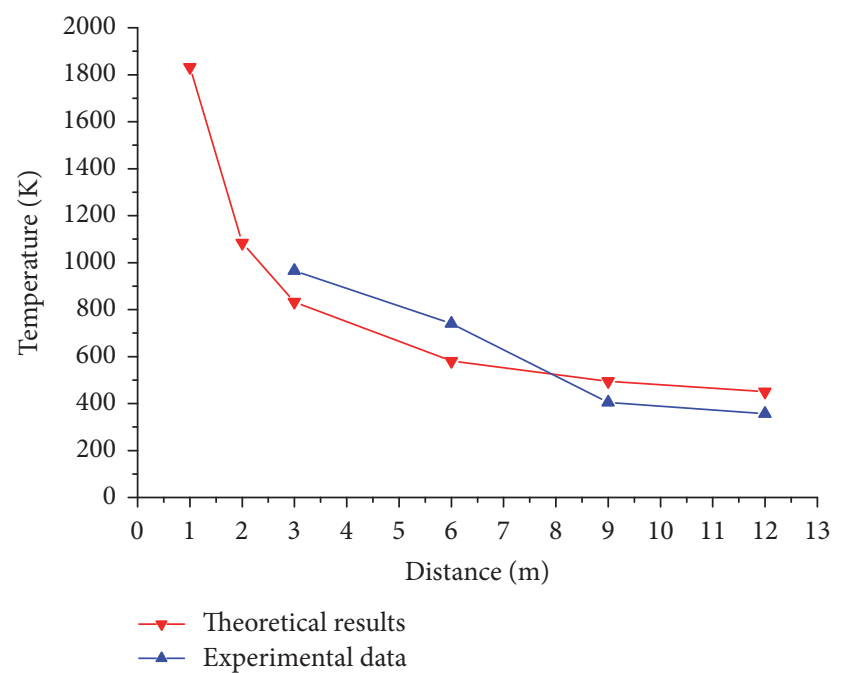

FIGURE 2: Comparison between theoretical calculations and experimental results for the temperature distribution.

3.2. Model Validation. To validate the accuracy and reliability of the model proposed in this paper, the air temperature distributions calculated with the above formula are compared with Yan et al.s experimental data [19]. Letting $m=8 \mathrm{~kg}, T_{0}=$ $298 \mathrm{~K}, P_{0}=101.325 \mathrm{kPa}, S=14.545 \mathrm{~m}^{2}, Q_{V}=7708 \mathrm{~kJ} / \mathrm{kg}$, and $\gamma=1.4$, the results are shown as Figure 2 .

Figure 2 demonstrates the consistency between the theoretical and experimental air temperature distributions in the tunnel, indicating that the trend of the air temperature distribution can be well reflected by the theoretical model. Therefore, the theoretical model proposed in this paper can be used to estimate the air temperature distribution in a tunnel after thermobaric explosion.

3.3. Temperature Distribution in Tunnel. The temperature distribution was calculated for a specific RDX-based TBX, which consists of $20 \% \mathrm{RDX}, 43 \%$ ammonium perchlorate (AP), 25\% Al, and 12\% hydroxyl-terminated polybutadiene (HTPB) in mass fraction, exploding at the tunnel entrance [26]. The parameters were set as $m=100 \mathrm{~kg}, Q_{V}=$ $3998 \mathrm{~kJ} / \mathrm{kg}$. Letting $T_{0}=298 \mathrm{~K}, P_{0}=101.325 \mathrm{kPa}, S=$ $4.32 \mathrm{~m}^{2}$, and $\gamma=1.4$, and the calculated curve of air temperature distribution shortly after the thermobaric explosion in the tunnel is illustrated in Figure 3.

The air temperature inside the tunnel decreases with the increasing distance, eventually reaching the original air temperature of the tunnel. In addition, the air temperature decreases rapidly in the range $0 \sim 100 \mathrm{~m}$ and then gradually becomes nearly constant, similarly to the attenuation of shock wave overpressure over an increasing distance.

\section{Dynamic Thermal Environment}

4.1. Numerical Model. A 2D tunnel model, which represents the longitudinal section along the central axis of the tunnel, was established in this paper as shown in Figure 4. Considering the computational cost, the model is limited to a 


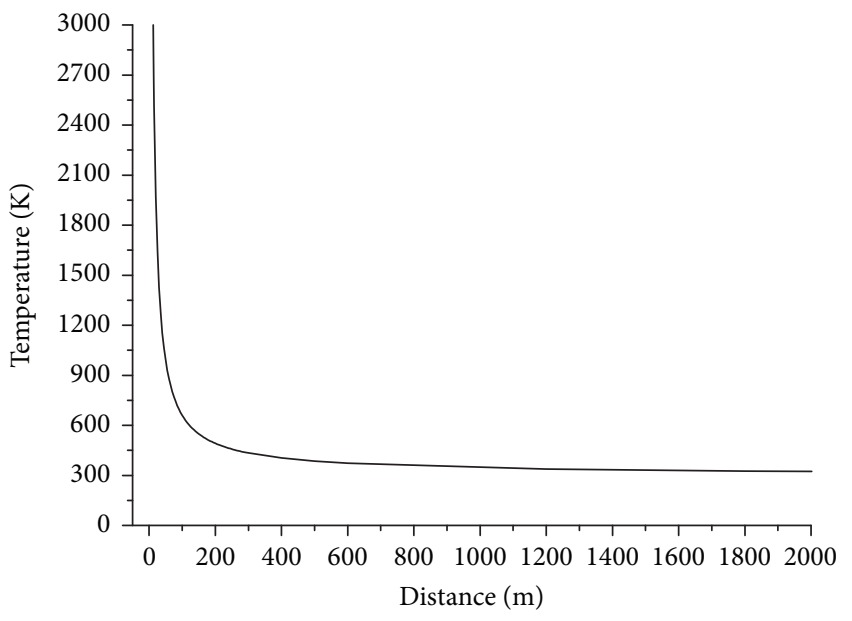

FIgURE 3: Calculated air temperature distribution in the tunnel.

$200 \mathrm{~m}$ long tunnel. In order to simulate the effects of the unmodeled section on results, the extrapolation method of flow field parameters was used at the end of the model [27]. The central part of the model represents the air domain with a height of $2.4 \mathrm{~m}$, and the upper and lower parts are the surrounding rocks, both with a height of $3 \mathrm{~m}$, which is greater than the thermal effective radius of the high-temperature air. The influence of gravity and buoyancy was taken into account.

4.2. Governing Equations. The continuity equation can be expressed as

$$
\frac{\partial \rho}{\partial t}+\operatorname{div}(\rho \mathbf{u})=0
$$

where $\rho$ is density; $t$ is time; $\mathbf{u}$ is the velocity vector of the fluid.

The momentum conservation equation can be expressed as

$$
\frac{\partial\left(\rho u_{i}\right)}{\partial t}+\operatorname{div}\left(\rho u_{i} \mathbf{u}\right)=\operatorname{div}\left(\mu \operatorname{grad} u_{i}\right)-\frac{\partial p}{\partial x_{i}}+S_{u_{i}},
$$

where $u_{i}$ represents the velocity components in the $x$ and $y$ directions; $\mu$ is the dynamic viscosity; $p$ is the pressure on the fluid microelement; $S_{u_{i}}$ represents the generalized source term in the $x$ and $y$ directions.

The energy conservation equation can be expressed as

$$
\frac{\partial(\rho T)}{\partial t}+\operatorname{div}(\rho \mathbf{u} T)=\operatorname{div}\left(\mu \frac{k}{c_{p}} \operatorname{grad} T\right)+S_{T},
$$

where $T$ is the fluid temperature; $k$ is the heat transfer coefficient of the fluid; $c_{p}$ is the constant-pressure specific heat; $S_{T}$ is the viscous dissipation term.

The air in tunnel is considered as still in the moment after explosion, and the ventilation system is stopped. The flow of high-temperature air is a low Reynolds number turbulent flow driven by the temperature difference. In order to characterize the airflow properly, the $k$ (turbulent kinetic energy) and $\varepsilon$ (turbulent dissipation rate) RNG model were utilized for its better performance. The equations of the model can be expressed as follows [28]:

$$
\begin{aligned}
\frac{\partial(\rho k)}{\partial t}+\frac{\partial\left(\rho k u_{i}\right)}{\partial x_{i}}= & \frac{\partial}{\partial x_{j}}\left(\alpha_{k} \mu_{\mathrm{eff}} \frac{\partial k}{\partial x_{j}}\right)+G_{k}+\rho \varepsilon \\
\frac{\partial(\rho \varepsilon)}{\partial t}+\frac{\partial\left(\rho \varepsilon u_{i}\right)}{\partial x_{i}}= & \frac{\partial}{\partial x_{j}}\left(\alpha_{\varepsilon} \mu_{\mathrm{eff}} \frac{\partial \varepsilon}{\partial x_{j}}\right)+\frac{C_{1 \varepsilon}^{*} \varepsilon}{k} G_{k} \\
& -C_{2 \varepsilon} \rho \frac{\varepsilon^{2}}{k}
\end{aligned}
$$

where $G_{k}=\mu_{t}\left(\partial u_{i} / \partial x_{j}+\partial u_{j} / \partial x_{i}\right)\left(\partial u_{i} / \partial x_{j}\right) ; \mu_{\text {eff }}=\mu+\mu_{t} ; \mu_{t}=$ $\rho C_{\mu}\left(k^{2} / \varepsilon\right) ; C_{\mu}=0.0845 ; \alpha_{k}=\alpha_{\varepsilon}=1.39 ; C_{1 \varepsilon}^{*}=C_{1 \varepsilon}-\eta(1-$ $\left.\eta / \eta_{0}\right) /\left(1+\beta \eta^{3}\right) ; C_{1 \varepsilon}=1.42, C_{2 \varepsilon}=1.68 ; \eta=\left(2 E_{i j} \cdot E_{i j}\right)^{1 / 2}(k / \varepsilon)$; $\eta_{0}=4.377, \beta=0.012 ; E_{i j}=(1 / 2)\left(\partial u_{i} / \partial x_{j}+\partial u_{j} / \partial x_{i}\right)$.

It is worth mentioning that the low Reynolds number turbulent flow simulated by the RNG $k-\varepsilon$ model, especially in the near-wall area, needs to match the appropriate wall function to get reasonable accuracy. Therefore, we used an enhanced wall treatment to complement the turbulence model in order to improve the accuracy in simulating the turbulent flow and the complicated near-wall flow field.

\subsection{Boundary and Initial Conditions}

4.3.1. Boundary Conditions. The above-mentioned equations are subject to the boundary conditions indicated by Figure 4 . The detailed boundary conditions for this problem were set as follows.

The far-field boundaries were set as no-slip solid walls with a constant temperature equivalent to the initial temperature of the surrounding rocks.

The two interfaces between separate blocks were considered as interior surfaces and set as temperature-coupled walls.

There are two kinds of boundary conditions for the tunnel entrance: one is the pressure outlet boundary, which was applied to the open tunnel case; the other is the wall boundary with third temperature boundary condition, which was applied to simulate the case in which the tunnel is blocked by fallen rocks. For the pressure outlet boundary, the return air temperature was set to $298 \mathrm{~K}$, ignoring the outdoor air temperature changes under the influence of the explosion. As for the wall boundary, the convective heat transfer coefficient was taken as $14.5 \mathrm{~W} / \mathrm{m}^{2} \cdot \mathrm{K}$ [29], and the free stream temperature was set to $298 \mathrm{~K}$.

4.3.2. Initial Conditions. The temperature distribution shortly after the explosion was assigned to the air field as the initial condition in the numerical model by a User Defined Function (UDF), which is a C language file that can dynamically connected to the FLUENT solver to improve its simulating performance. It is worth noting that the calculated temperature is relatively high in the vicinity of the explosion source. We therefore made a correction by setting the fireball temperature $(3153.3 \mathrm{~K})$ as the air temperature at the explosion source and connecting to the theoretical curve in $3000 \mathrm{~K}$ by linear 


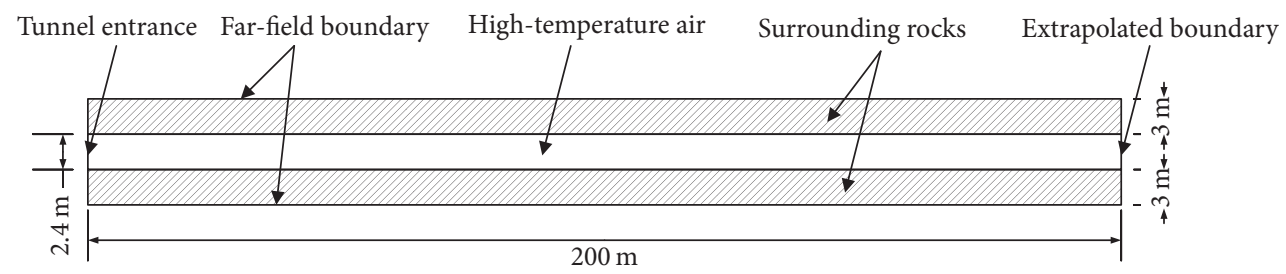

FIGURE 4: Schematic illustration of the numerical model.

TABLE 2: Physical properties of surrounding rocks.

\begin{tabular}{lcc}
\hline $\begin{array}{l}\text { Density } \\
\rho_{r}\left(\mathrm{~kg} / \mathrm{m}^{3}\right)\end{array}$ & $\begin{array}{c}\text { Constant-pressure } \\
\text { specific heat } \\
c_{p r}(\mathrm{~J} / \mathrm{kg} \cdot \mathrm{K})\end{array}$ & $\begin{array}{c}\text { Heat conductivity } \\
\text { coefficient } \\
\lambda_{r}(\mathrm{~W} / \mathrm{m} \cdot \mathrm{K})\end{array}$ \\
\hline 2500 & 840 & 2.04 \\
\hline
\end{tabular}

attenuation. Considering the radiation heat transfer between the high-temperature air and the surrounding objects, the Discrete Transfer Radiation Model (DTRM) was selected.

The surrounding rock was considered to be homogeneous and isotropic, and its initial temperature was set to $293 \mathrm{~K}$.

4.4. Material Physical Properties. The physical properties of the surrounding rock are shown in Table 2.

For the high-temperature air, the main physical parameters of the high-temperature air in the tunnel vary significantly with temperature, according to the following empirical piecewise polynomial functions [30]:

(1) Density

$273 \mathrm{~K} \leq T<900 \mathrm{~K}$,

$$
\begin{aligned}
\rho_{a}= & -5.137 \times 10^{-19} T^{7}+2.198 \times 10^{-15} T^{6}-3.907 \\
& \times 10^{-12} T^{5}+3.738 \times 10^{-9} T^{4}-2.084 \times 10^{-6} T^{3} \\
& +6.85 \times 10^{-4} T^{2}-0.128 T+12.148
\end{aligned}
$$

$900 \mathrm{~K} \leq T<3500 \mathrm{~K}$,

$$
\begin{aligned}
\rho_{a}= & 4.612 \times 10^{-14} T^{4}-3.888 \times 10^{-10} T^{3}+1.266 \\
& \times 10^{-6} T^{2}-1.99 \times 10^{-3} T+1.503012
\end{aligned}
$$

(2) Constant-pressure specific heat

$$
\begin{aligned}
c_{a_{q}}= & -1.449 \times 10^{-11} T^{4}+1.363 \times 10^{-7} T^{3}-5.06 \\
& \times 10^{-4} T^{2}+0.906 T+689.019
\end{aligned}
$$

(3) Heat conductivity coefficient

$$
\lambda_{a}=6.714 \times 10^{-5} T+0.00144
$$

(4) Radiation absorption coefficient

$$
\begin{aligned}
273 \mathrm{~K} \leq T<1173 \mathrm{~K}, \\
\alpha_{a}=-1.29968 \times 10^{-7} T^{2}+2.95398 \times 10^{-4} T \\
\quad-0.03899
\end{aligned}
$$

$$
1173 \mathrm{~K} \leq T<3500 \mathrm{~K} \text {, }
$$

$$
\begin{aligned}
\alpha_{a}= & -3.38463 \times 10^{-9} T^{2}-5.04093 \times 10^{-4} T \\
& +0.19361
\end{aligned}
$$

In addition, the air viscosity can be calculated by the following equation [31]:

$$
\frac{\mu}{\mu_{0}}=\left(\frac{T}{T_{0}}\right)^{3 / 2} \times \frac{T_{0}+B}{T+B},
$$

where $\mu_{0}=0.18 \times 10^{-4} \mathrm{~Pa} \cdot \mathrm{s}, T_{0}=298 \mathrm{~K}$, and $B=110.4$.

4.5. Solution Scheme and Procedure. A quadrilateral mesh was applied to the model and appropriately refined at the fluid-solid coupling region, amounting to 44000 mesh. The governing equations were discretized using the finite volume method and solved by the SIMPLE algorithm combining the conditions mentioned above. All the presented numerical results were calculated by using the FLUENT software with a time step of $0.1 \mathrm{~s}$. The convergence was reached when residuals were smaller than $10^{-6}$ for energy and $10^{-3}$ for other variables.

\subsection{Results and Discussion}

4.6.1. Open Tunnel Case. Figure 5 shows the temporal and spatial variation of temperature in the open tunnel case after the explosion of $100 \mathrm{~kg}$ TBX at the tunnel portal. During the preliminary period, high-temperature air near the explosion source flows towards the top of the tunnel due to the action of the buoyancy force and then forms a ceiling jet. The entrainment of such jet causes the cold air outside and the cooler air in the depth of the tunnel to flow along the bottom of the tunnel towards the explosion source in a natural convection process with thermal stratification. As time elapses, the high-temperature region gradually concentrates on the top and moves deep into the tunnel. This is probably due to the difference in driving forces caused by the unbalanced temperature difference on both sides of the high-temperature region and to the cooling effect of the air outside the tunnel. 


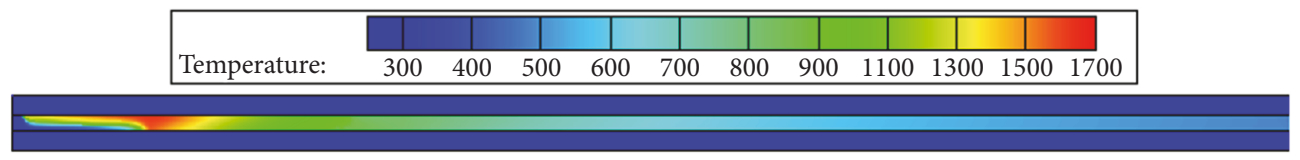

(a) $6 \mathrm{~s}$

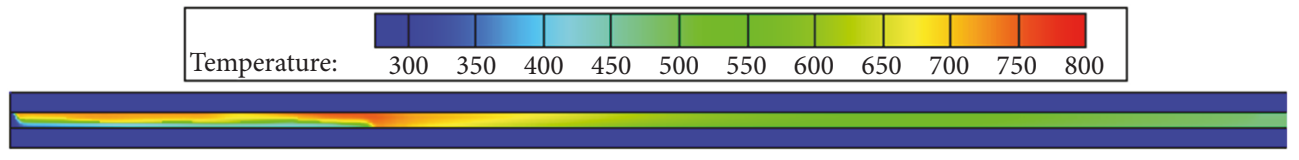

(b) $24 \mathrm{~s}$

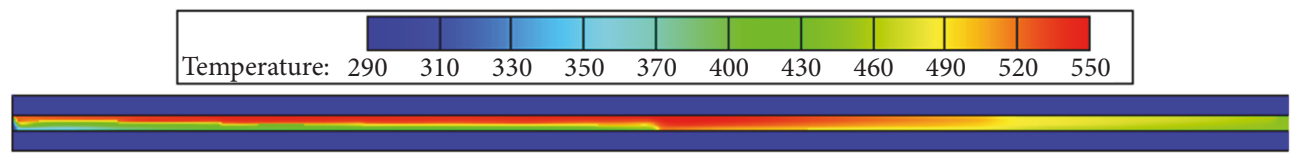

(c) $60 \mathrm{~s}$

FIGURE 5: Temporal and spatial variation of temperature in the open tunnel.

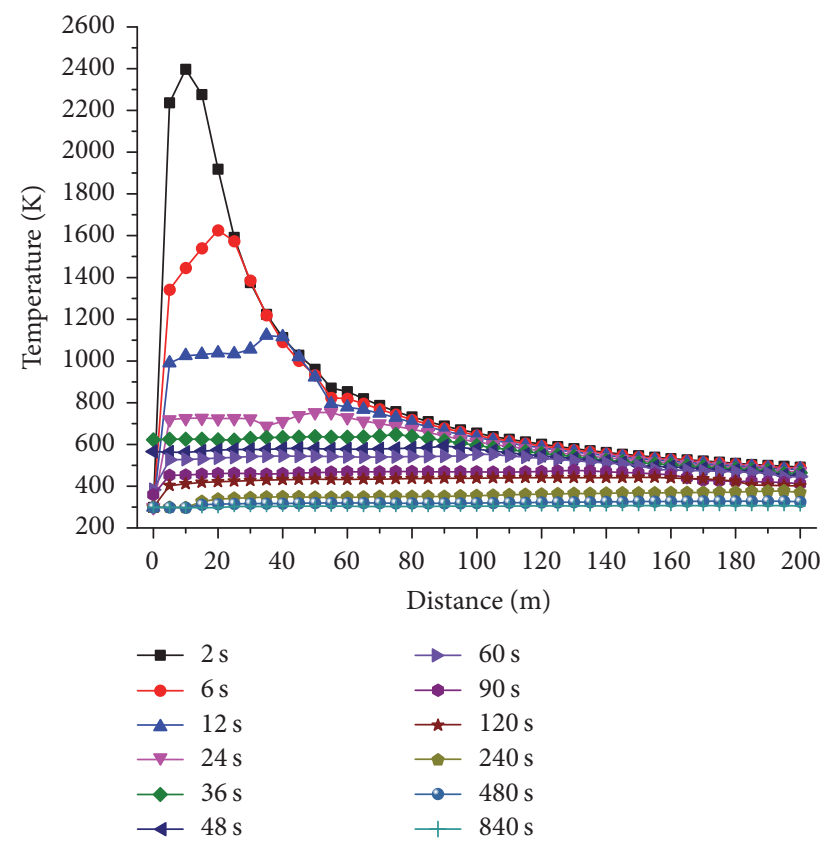

FIGURE 6: Spatial distribution of temperature in the open tunnel at different times.

In order to achieve a more accurate understanding of the temporal and spatial distribution of the air temperature inside the tunnel, Figure 6 presents the curve of maximum air temperature measured at a height of $20 \mathrm{~cm}$ below the tunnel ceiling. The high-temperature region shows a very wide range, and its displacement and attenuation are easily observed at times until 240 seconds. Furthermore, the temperature curves of $240 \mathrm{~s}, 480 \mathrm{~s}$, and $840 \mathrm{~s}$ are almost coincident, which means that the relatively high temperature of $308 \sim 348 \mathrm{~K}$ is sustained for a long time after the explosion. This phenomenon is mainly due to the high content of aluminum powder as thermite in the TBX. When the explosive explodes, the thermite flies and deflagrates, eventually forming a high-temperature cloud with a wide range, showing volume explosion characteristics. Since the surrounding air has received the heat generated by the explosion and after-burning of TBX, on the one hand, the explosion temperature will be higher; on the other hand, the high-temperature duration will increase.

4.6.2. Blocked Tunnel Case. The temporal and spatial variations of temperature in the blocked tunnel case are illustrated in Figure 7. Unlike the open tunnel case, the hightemperature air can only flow deep into the tunnel along the ceiling due to the blocked entrance in this case, entraining the cooler air from the depth of the tunnel and establishing a one-sided natural convection. As a major difference between the two cases, the high-temperature region is concentrated near the explosion source in the blocked tunnel, stretching gradually over time, but without a clear displacement. This difference could be attributed to the shortage of cold air from the outside. Another difference lies in the disappearing of thermal stratification at $300 \mathrm{~s}$ in the blocked tunnel case, with the temperature distribution in the longitudinal section of the tunnel becoming nearly uniform at later times. One reason for this could be that the entrained cooler airflow will head back when it is blocked by the fallen rock and then mixes and exchanges heat with the hot air favoring the formation of a uniform temperature field.

Figure 8 shows the curve of maximum air temperature measured $20 \mathrm{~cm}$ below the tunnel ceiling in the blocked tunnel case. The peak temperature is always found at the second gauging point from the entrance, which is affected by the cold rock surface to a lower extent than the first one. Moreover, the relatively high-temperature region is always concentrated within $100 \mathrm{~m}$ of the explosion source, attenuating over time. Compared with Figure 6, the air temperature inside the tunnel in the blocked case decreases more slowly, which means that the probability of causing a secondary fire disaster is much higher. Therefore, more attention should be 
(a) $16 \mathrm{~s}$

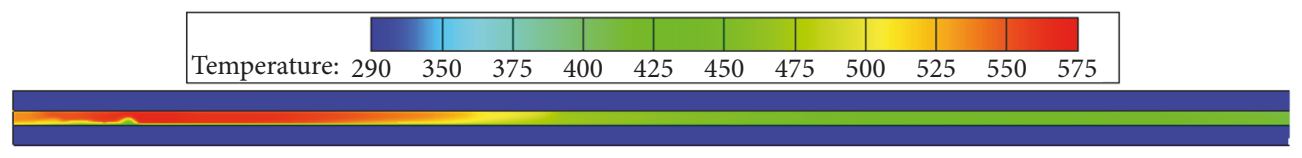

(b) $84 \mathrm{~s}$

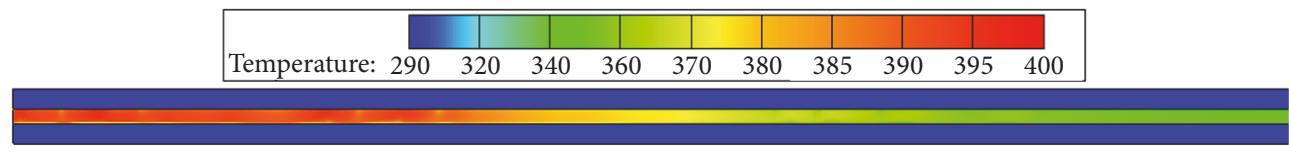

(c) $300 \mathrm{~s}$

FIgURE 7: Temporal and spatial variation of temperature in the blocked tunnel.

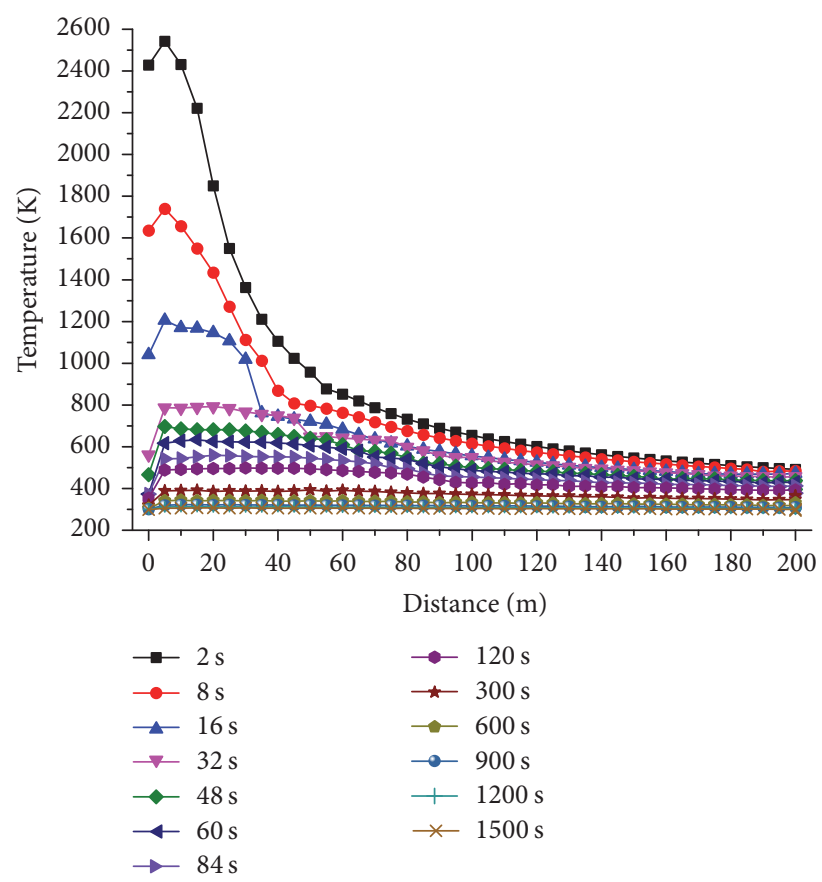

FIGURE 8: Spatial distribution of temperature in the blocked tunnel at different times.

paid to the thermal protection design of protective works, especially in the tunnel portal.

4.6.3. Influence of Explosive Mass. In order to study the influence of TBX mass on the dynamic thermal environment changes, three TBXs of the same type with different mass $(1 \mathrm{~kg}, 10 \mathrm{~kg}$, and $100 \mathrm{~kg}$, resp.) were chosen as the research subjects. Keeping other parameters at the same value as in the previous paragraphs, the maximum temperature attenuation curves for different explosive masses were obtained in both the open and blocked tunnel cases, and they are shown in Figure 9. The curves indicate that increasing the explosive mass significantly increases the high-temperature duration
TABLE 3: Annual average ground temperature of typical cities [32].

\begin{tabular}{lc}
\hline City & Annual average ground temperature (K) \\
\hline Shenyang & 283.47 \\
Beijing & 287.11 \\
Wuhan & 291.94 \\
Guangzhou & 296.76 \\
\hline
\end{tabular}

and decreases the decay rate. Taking the open tunnel case as an example, the cooling times (which are defined as the times when the maximum temperature in the tunnel is reduced to $308 \mathrm{~K}$ ) are $161.5 \mathrm{~s}, 439.4 \mathrm{~s}$, and $724.8 \mathrm{~s}$ corresponding to the explosive with $1 \mathrm{~kg}, 10 \mathrm{~kg}$, and $100 \mathrm{~kg}$, respectively. This mainly happens because a greater TBX mass releases a larger amount of energy when it explodes, thus requiring a longer time to cool down. Furthermore, the cooling times of $1 \mathrm{~kg}$, $10 \mathrm{~kg}$, and $100 \mathrm{~kg}$ TBXs in the blocked tunnel are 1.56, 1.63, and 1.72 times as long as the open tunnel case, respectively. This indicates that a larger amount of explosive makes TBX more powerful in the blocked tunnel.

4.6.4. Influence of Initial Ground Temperature. In order to study the influence of initial temperature conditions on the dynamic thermal environment changes, the different annual average ground temperatures of four typical Chinese cities with totally different climatic conditions were chosen as the initial temperature of the surrounding rocks, as shown in Table 3.

Setting the TBX mass as $100 \mathrm{~kg}$ and keeping other parameters consistent with the previous text, the maximum temperature attenuation curves for different initial ground temperatures were obtained in both the open and blocked tunnel cases and they are shown in Figure 10. In both cases, a higher initial ground temperature results in a slower attenuation, and the temperature difference between different conditions grows larger over time. Taking the blocked tunnel case as an example, the highest air temperature in the tunnel in the four conditions at $600 \mathrm{~s}$ is $336.42 \mathrm{~K}, 339.99 \mathrm{~K}$, 


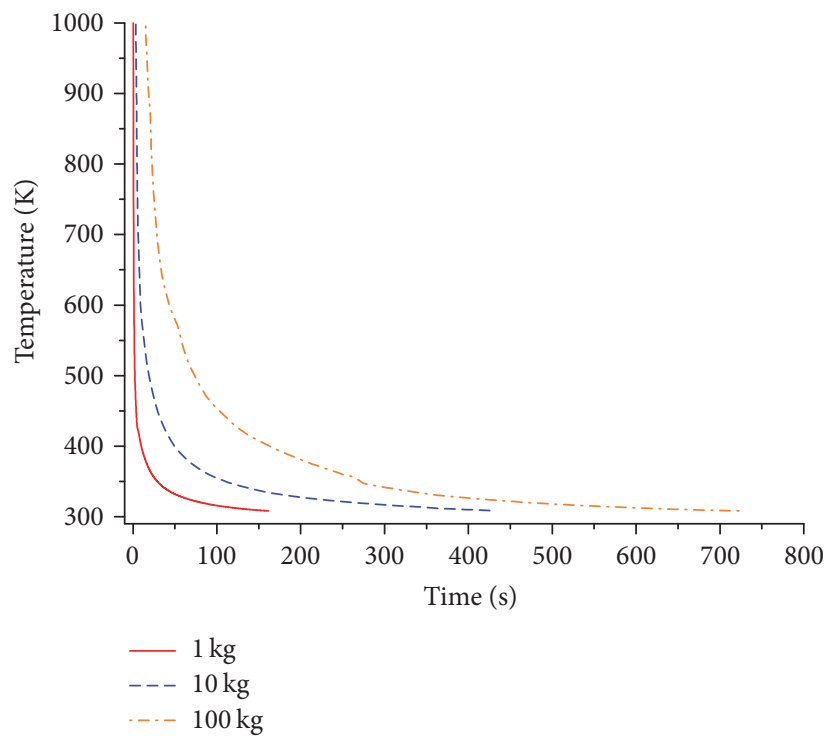

(a) Open tunnel

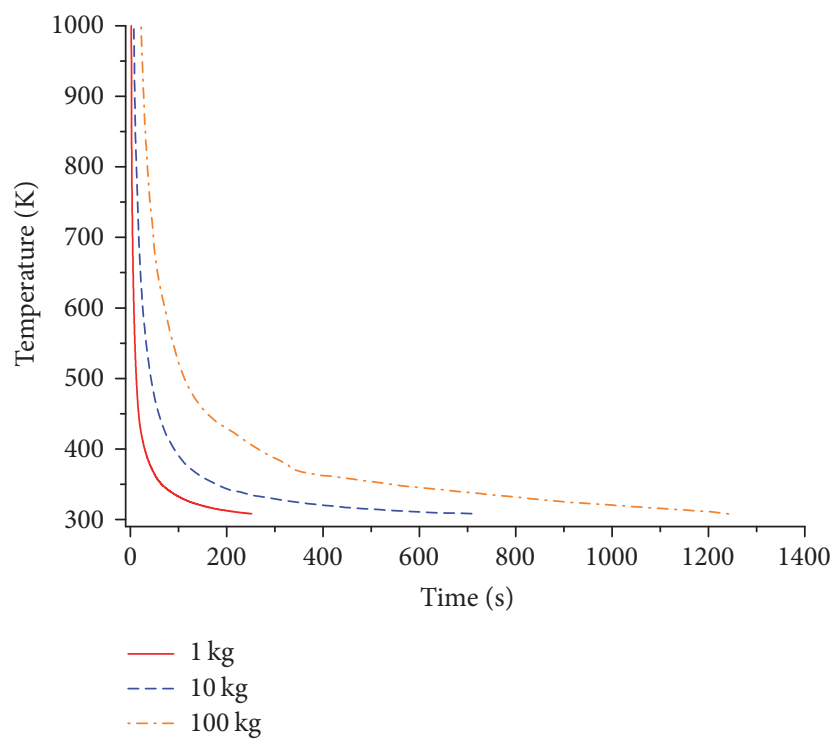

(b) Blocked tunnel

FIGURE 9: Maximum temperature attenuation curves of different TBX mass.

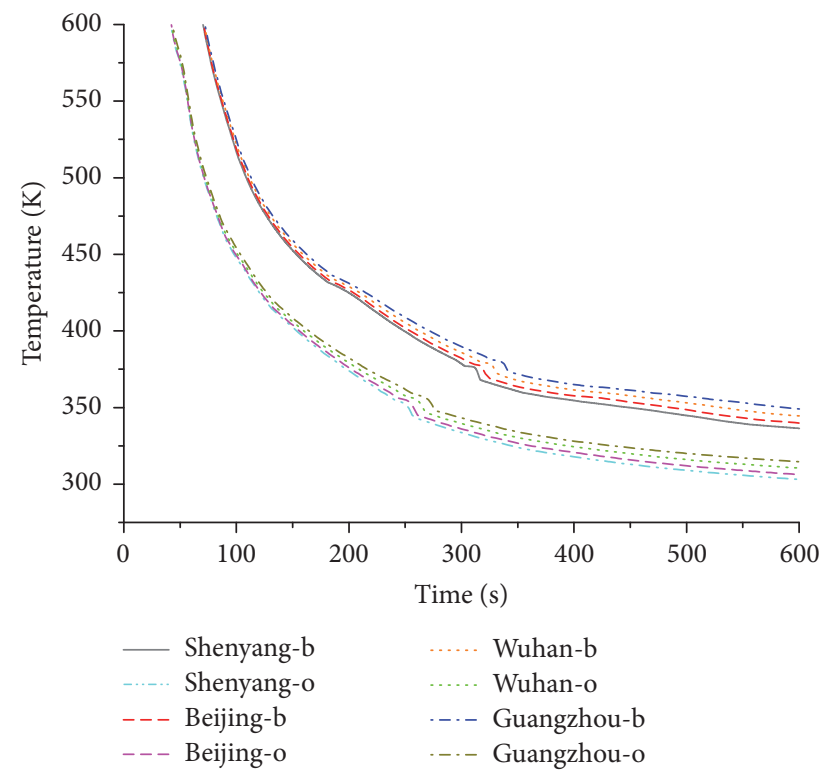

FIGURE 10: Maximum temperature attenuation curves of different initial ground temperature.

$344.55 \mathrm{~K}$, and $349.07 \mathrm{~K}$ while the values at $100 \mathrm{~s}$ were $517.05 \mathrm{~K}$, $519.29 \mathrm{~K}, 522.03 \mathrm{~K}$, and $524.99 \mathrm{~K}$. Thus, it is possible to state that a higher initial ground temperature extends the hightemperature duration of TBX.

In order to investigate the extent to which the initial temperature conditions affect the thermal environment changes in different tunnel cases, the cooling time can be taken as an index and an influence rate parameter can be defined as

$$
\xi=\frac{\Delta t_{\text {cooling }}}{\Delta F / \Delta F_{\max }},
$$

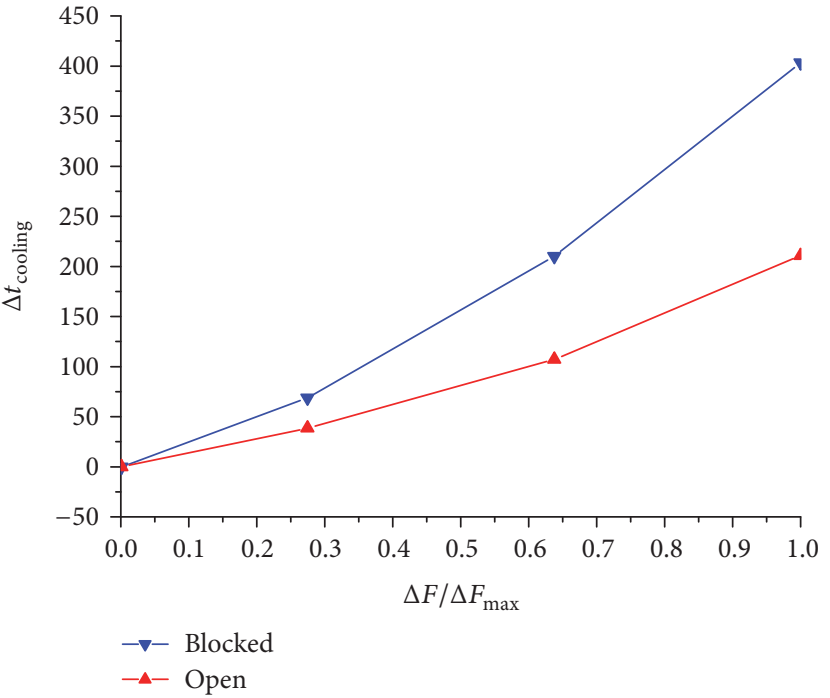

FIGURE 11: Cooling time changes against initial ground temperature.

where $\Delta t_{\text {cooling }}$ is the change in cooling time caused by the initial ground temperature change $\Delta F$, and $\Delta F_{\max }$ is the maximum change in the initial ground temperature.

Figure 11 shows the relationship between the change in cooling time and the change in initial ground temperature for the two different tunnel cases. The curve indicates that the cooling time increases exponentially with increasing change rate of initial ground temperature, and the influence rate (i.e., the slope of the curve) of the blocked tunnel case is larger than that of the open tunnel case at all time. In other words, the increase of initial ground temperature favors the hightemperature performance of TBX, especially for the blocked tunnel. 


\section{Conclusions}

To learn about the thermal environment inside a tunnel after thermobaric explosion in detail, a damage evaluation method for thermal radiation of explosion fireball in a tunnel was established based on the Baker fireball model and the heat dose damage criterion; the air temperature distribution inside a tunnel shortly after the explosion was studied by theoretical derivation; taking the calculated distribution as an initial condition, the dynamic thermal environment after the explosion was analyzed in two different cases (open tunnel or blocked tunnel) and in different conditions (TBX mass or initial ground temperature) was numerically analyzed. Based on the above investigations, the following conclusions may be drawn:

(1) The fireball thermal radiation damage ranges for $100 \mathrm{~kg}$ of RDX-based TBX in a tunnel were determined based on the damage evaluation method. The thermal radiation lethal range, serious injury range, and slight injury range are $39.77 \mathrm{~m}, 48.88 \mathrm{~m}$, and $73.80 \mathrm{~m}$, respectively. The thermal radiation damage occurs mainly in the vicinity of the explosion source.

(2) The air temperature inside a tunnel shortly after explosion decreases continuously with the increase of distance from the explosion source and finally reaches the initial air temperature. In the calculation condition of this paper, the air temperature decreases rapidly in the range $0 \sim 100 \mathrm{~m}$ and then gradually becomes nearly constant.

(3) In the open tunnel case, the high-temperature region gradually concentrates on the top and moves towards the depth of the tunnel. Conversely, in the blocked tunnel case, the high-temperature region concentrates near the explosion source without a clear displacement and with a shorter duration of thermal stratification, stretching gradually over time. The decay rate of air temperature inside a tunnel is slower in the blocked case, which increases the probability of causing a secondary fire disaster.

(4) Increasing the explosive mass significantly increases the high-temperature duration and decreases the decay rate. The cooling time of different TBXs masses in the blocked tunnel is $1.56,1.63$, and 1.72 times as long as the open tunnel, showing that a larger amount of explosive makes TBX more powerful in the blocked tunnel.

(5) In both the open and closed tunnel cases, a higher initial ground temperature results in a slower attenuation, and the temperature differences between different conditions are larger and larger over time. The cooling time increases exponentially with increasing change rate of initial ground temperature, and the influence rate of the blocked tunnel case is larger than that of the open tunnel case at all times, showing that the increase of the initial ground temperature favors the high-temperature performance of TBX, especially for the blocked tunnel.

\section{Conflicts of Interest}

The authors confirm that this paper's content has no conflicts of interest.

\section{References}

[1] W. A. Trzciński and L. Maiz, "Thermobaric and enhanced blast explosives - properties and testing methods," Propellants, Explosives, Pyrotechnics, vol. 40, no. 5, pp. 632-644, 2015.

[2] X. Li, C. Miao, Q. Wang, and Z. Geng, "Antiknock performance of interlayered high-damping-rubber blast door under thermobaric shock wave," Shock and Vibration, vol. 2016, Article ID 2420893, 9 pages, 2016.

[3] X. L. Xing, S. X. Zhao, Z. Y. Wang, and G. T. Ge, "Discussions on thermobaric explosives (TBXs)," Propellants, Explosives, Pyrotechnics, vol. 39, no. 1, pp. 14-17, 2014.

[4] E. W. Anna, "Aspects of thermobaric weaponry," Military Technology, no. 4, pp. 3-6, 2003.

[5] P. Dearden, "New blast weapons.," Journal of the Royal Army Medical Corps, vol. 147, no. 1, pp. 80-86, 2001.

[6] A. L. Kuhl, J. B. Bell, V. E. Beckner et al., "Numerical simulation of thermobaric explosions," in Proceedings of the 38th Int. Conference of ICT Energetic Materials, FRG, Karlsruhe, Germany, 2007.

[7] H. Reichenbach, P. Neuwald, and A. L. Kuhl, "Waveforms measured in confiend thermabaric explosions," in Proceedings of the 38th Int. Conference of ICT Energetic Materials, FRG, Karlsruhe, Germany, 2007.

[8] W. A. Trzciński and K. Barcz, "Investigation of blast wave characteristics for layered thermobaric charges," Shock Waves, vol. 22, no. 2, pp. 119-127, 2012.

[9] W. A. Trzciński, K. Barcz, J. Paszula, and S. Cudziło, "Investigation of blast performance and solid residues for layered thermobaric charges," Propellants, Explosives, Pyrotechnics, vol. 39, no. 1, pp. 40-50, 2014.

[10] D. Simić, M. Popović, R. Sirovatka et al., "Influence of cast composite thermobaric explosive compositions on air shock wave parameters," Scientific Technical Review, vol. 23, no. 2, pp. 6369, 2013.

[11] Y. L. Zhang, H. B. Zhai, Z. R. Li et al., "experimental research on the contrast of the fireballs surface temperature of TNT and thermobaric explosive," Explosive Materials, vol. 44, no. 5, pp. 23-26, 2015 (Chinese).

[12] A. K. Mohamed, H. E. Mostafa, and S. Elbasuney, "Nanoscopic fuel-rich thermobaric formulations: chemical composition optimization and sustained secondary combustion shock wave modulation," Journal of Hazardous Materials, vol. 301, pp. 492503, 2016.

[13] Q.-L. Yan, W. A. Trzciński, S. Cudziło et al., “Thermobaric effects formed by aluminum foils enveloping cylindrical charges," Combustion and Flame, no. 166, pp. 148-157, 2015.

[14] X.-L. Li and J.-M. Hui, "Detonation temperature of thermobaric explosives," Explosion and Shock Waves, vol. 28, no. 5, pp. 471475, 2008 (Chinese).

[15] X. Y. Guo, B. Li, L. J. Wang et al., "Measurement of blast temperature field and study of thermal radiation effect for thermo-baric explosive," Journal of Projectiles, Rockets, Missiles and Guidance, vol. 28, no. 5, pp. 119-121, 2008 (Chinese).

[16] B. Li, X.-Y. Guo, and L.-F. Xie, "Study on thermal sustaining damage ability of thermobaric explosive," Journal of Ballistics, vol. 21, no. 4, pp. 99-102, 2009. 
[17] Q. Zhong, B.-L. Wang, J. Huang, Y.-Y. Fang, and J.-M. Hui, "Application of a dynamic model to thermal damage estimation of thermobaric explosives," Explosion and Shock Waves, vol. 31, no. 5, pp. 528-532, 2011 (Chinese).

[18] J. J. Yan, P. G. Jin, H. B. Li et al., "Experimental investigation of thermobaric explosive afterburn effect in finite space," Science Technology and Engineering, vol. 17, no. 15, pp. 154-157, 2015 (Chinese).

[19] X. M. Yan, J. J. Su, Z. R. Li et al., "Experimental study on explosive thermal effect of thermal-baric explosives in tunnel," Initiators \& Pyrotechnics, no. 1, pp. 22-25, 2015 (Chinese).

[20] S. B. Dorofeev, V. P. Sidorov, A. A. Efimenko et al., "Fireballs from deflagration and detonation of heterogeneous fuel-rich clouds," Fire Safety Journal, vol. 25, no. 4, pp. 323-336, 1995.

[21] W. E. Baker, P. A. Cox, P. S. Westine et al., Explosion Hazards and Evaluation, Elsevier Science Pub. Co., New York, NY, USA, 1983.

[22] W. E. Martinsen and J. D. Marx, "An improved model for the prediction of radiant heat from fireballs," in Proceedings of the International Conference and Workshop on Modeling Consequences of Accidental Releases of Hazardous Materials, pp. 1-16, San Francisco, Calif, USA, 1999.

[23] C. H. Bai, H. M. Liang, J. P. Li et al., Cloud Detonation, Science Press, Beijing, China, 2012.

[24] A. M. Benselama, M. J.-P. William-Louis, F. Monnoyer, and C. Proust, "A numerical study of the evolution of the blast wave shape in tunnels," Journal of Hazardous Materials, vol. 181, no. 1-3, pp. 609-616, 2010.

[25] L. Chen, X. P. Long, C. G. Feng et al., Detonation of Explosives Containing Aluminum, National Defense Industry Press, Beijing, China, 2004.

[26] J. F. Mao, F. Chen, and P. M. Hou, "Study on the shock wave damage effect of thermobaric explosive explosion in tunnel entrance," Chinese Quarterly of Mechanics, vol. 37, no. 1, pp. 184193, 2016 (Chinese).

[27] S. Z. Yang, Research on Propagation and Injury Model of Coal Dust Explosion in Confined Space, Henan Polytechnic University, Jiaozuo, China, 2010.

[28] ANSYS, Inc., ANSYS FLUENT Theory Guide (Release 14.0), ANSYS, Inc., Canonsburg, PA, USA, 2011.

[29] Y. L. Duan, X. Q. Zhou, H. Y. Wang et al., "Analysis of convective heat transfer coefficient in roadway under mine gas explosion," Coal Mine Safety, vol. 431, no. 2, pp. 105-108, 2010 (Chinese).

[30] ANSYS, Inc., ANSYS FLUENT Tutorial Guide (Release 14.0), ANSYS, Inc., Canonsburg, PA, USA, 2011.

[31] J. L. Yu, X. Q. Yan, and L. Chen, "Experiment and numerical simulation study on aluminum dust explosion in confined space," Industrial Safety and Environmental Protection, vol. 37, no. 11, pp. 12-15, 2011 (Chinese).

[32] H. Z. Zhang, X. Z. Shi, D. S. Yu et al., "Spatial prediction of soil temperature in China," Acta Pedologica Sinica, vol. 46, no. 1, pp. 1-8, 2009 (Chinese). 


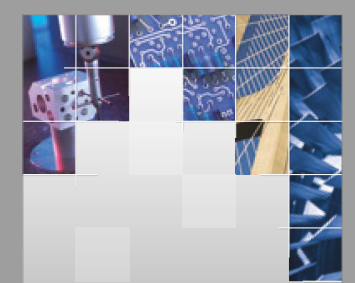

\section{Enfincering}
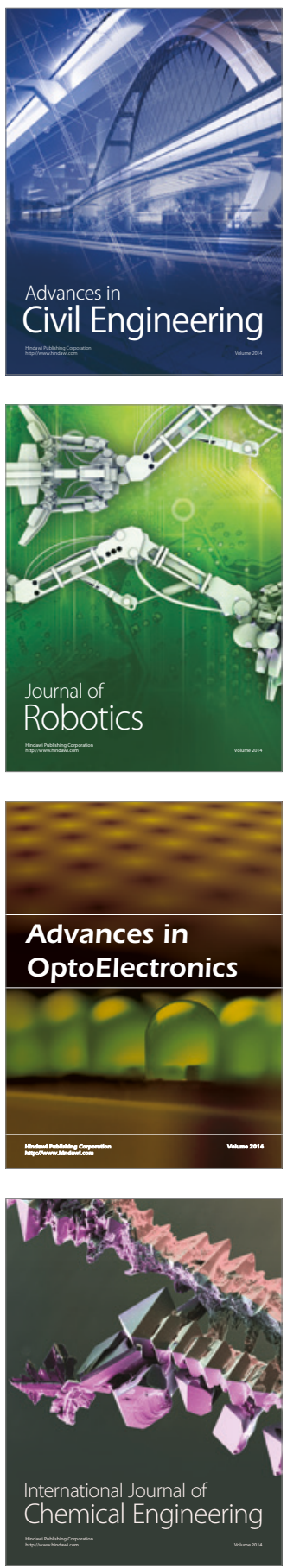

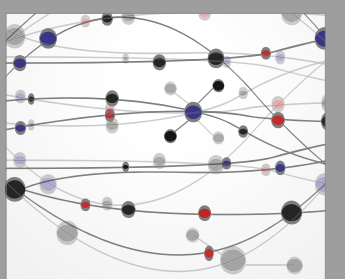

The Scientific World Journal

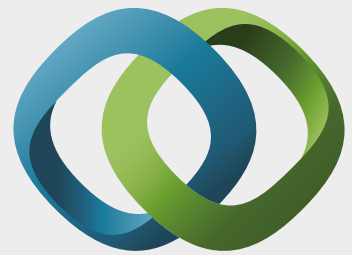

\section{Hindawi}

Submit your manuscripts at

https://www.hindawi.com
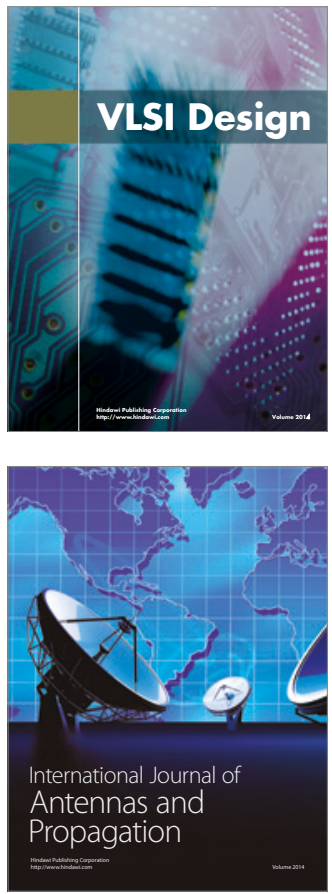

\section{Rotating}

Machinery
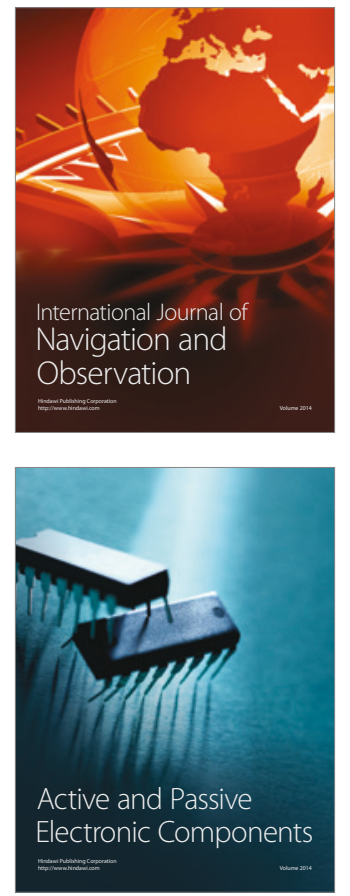
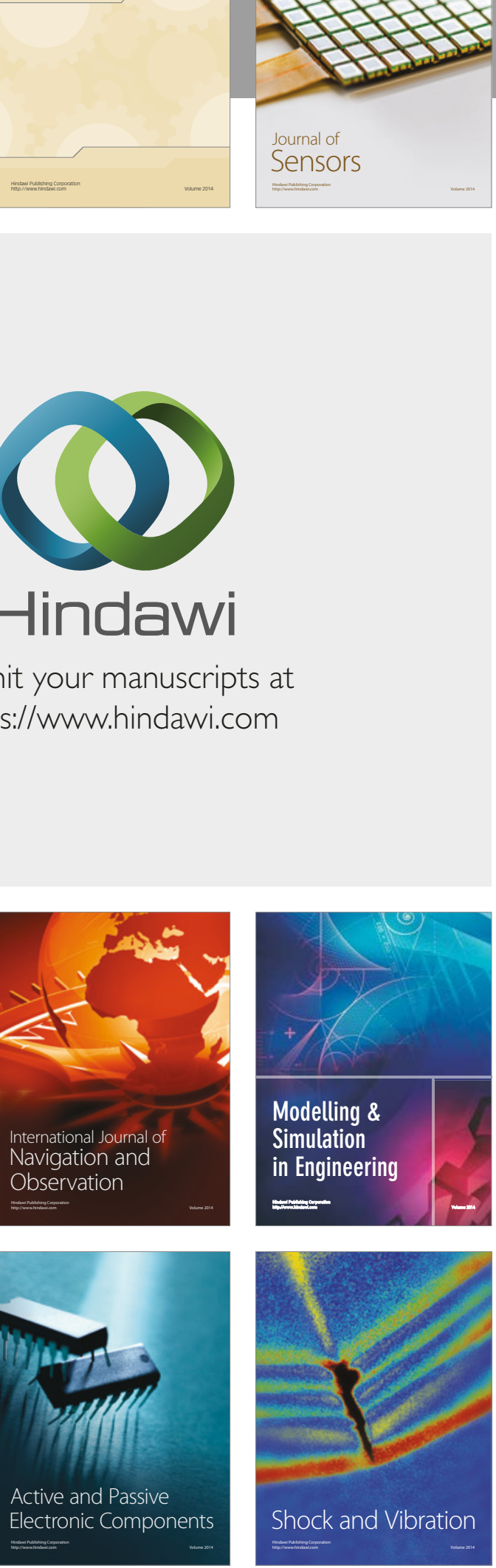
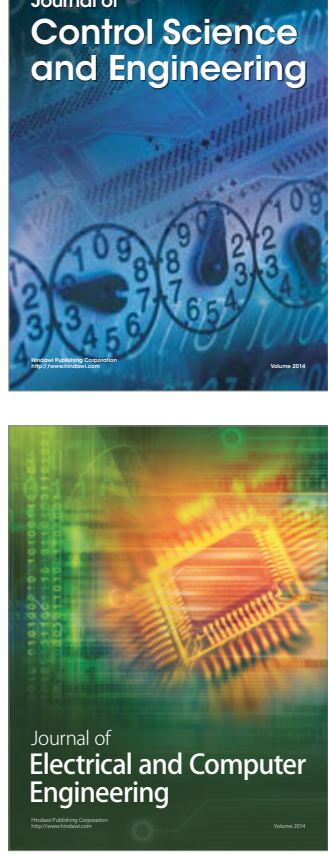

Distributed

Journal of

Control Science

and Engineering
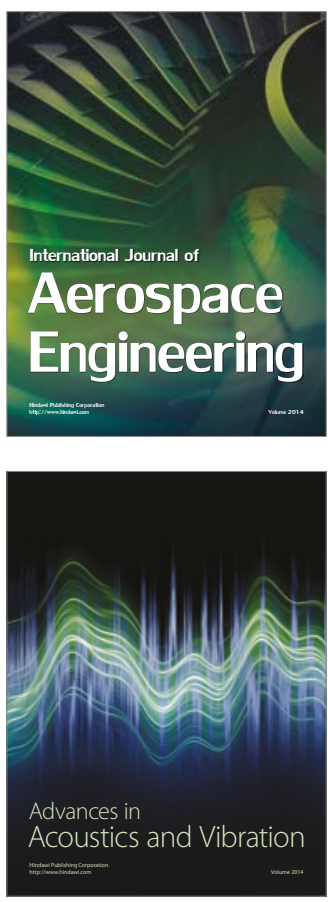

Sensor Networks 